\title{
In Vitro Culture and Characterization of Human Lung Cancer Circulating Tumor Cells Isolated by Size Exclusion from an Orthotopic Nude-Mouse Model Expressing Fluorescent Protein
}

\author{
Katarina Kolostova • Yong Zhang • Robert M. Hoffman • \\ Vladimir Bobek
}

Received: 27 May 2014 / Accepted: 11 August 2014 / Published online: 22 August 2014

(C) The Author(s) 2014. This article is published with open access at Springerlink.com

\begin{abstract}
In the present study, we demonstrate an animal model and recently introduced size-based exclusion method for circulating tumor cells (CTCs) isolation. The methodology enables subsequent in vitro CTC-culture and characterization. Human lung cancer cell line H460, expressing red fluorescent protein (H460-RFP), was orthotopically implanted in nude mice. CTCs were isolated by a size-based filtration method and successfully cultured in vitro on the separating membrane (MetaCell $\left.{ }^{\circledR}\right)$, analyzed by means of time-lapse imaging. The cultured CTCs were heterogeneous in size and morphology even though they originated from a single tumor. The outer CTC-membranes were blebbing in general. Abnormal mitosis resulting in three daughter cells was frequently observed. The expression of RFP ensured that the CTCs originated from lung tumor. These readily isolatable, identifiable and cultivable
\end{abstract}

K. Kolostova $\cdot$ V. Bobek $(\bowtie)$

Department of Laboratory Genetics, University Hospital Kralovske

Vinohrady, Srobarova 50, 10034 Prague, Czech Republic

e-mail: vbobek@centrum.cz

Y. Zhang • R. M. Hoffman

AntiCancer, Inc., San Diego, CA, USA

R. M. Hoffman

Department of Surgery, University of California, San Diego, CA, USA

\section{Bobek}

3rd Department of Surgery First Faculty of Medicine, Charles University in Prague and University Hospital Motol, Prague, Czech Republic

V. Bobek

Department of Histology and Embryology, Wroclaw Medical

University, Wroclaw, Poland
CTCs can be used to characterize individual patient cancers and for screening of more effective treatment.

Keywords Lung cancer - Orthotopic · Circulating tumor cells $\cdot$ CTC $\cdot$ In vitro culture $\cdot$ CTC $\cdot$ MetaCell $\cdot$ Filtration $\cdot$ Size $\cdot$ Fluorescence $\cdot$ RFP

\section{Introduction}

The detection of circulating tumour cells (CTCs) in the peripheral blood of patients with solid epithelial tumors holds a great promise, and many exciting separation technologies have been developed over the past years [1].

However, detecting CTCs remain technically challenging. CTCs are present at very low concentrations of one tumor cell in the background of millions of blood cells. Extremely sensitive methods are required for their identification and characterization. Nevertheless, presence of CTCs could be an evidence for disease progress towards cancer dissemination $[2,3]$. However, the role of CTCs as a disease marker may be unique under different clinical conditions and should be carefully interpreted. Clinical validation of these new biomarkers requires analysis of CTCs throughout the course of in vivo and in vitro studies.

Animal model studies using orthotopic metastatic models could help to identify the role of CTCs as potential biomarker, enabling mutational analysis and functional testing of metastasis expansion from CTCs.

In the present study, we demonstrate a method for CTC isolation based on size exclusion from an orthotopic nude mouse model of human lung cancer labeled with RFP (red fluorescent protein), using capillary-action-driven blood flow though porous membranes. The separating membranes can be 
used for in vitro culture of the CTCs immediately after CTCsenrichment process.

\section{Materials and Methods}

Cell Culture The human H460 lung cancer cell line, expressing RFP, used in this study has been described previously [4]. The cells were grown in RPMI-1640 medium supplemented with $10 \%$ fetal bovine serum (FBS) and gentamicin (Life Technologies, Carlsbad, CA) to 70-80\% confluence as described previously [5].

Orthotopic Model of H460-RFP in Nude Mice A subcutaneously-growing H460-RFP tumor fragment $\left(1 \mathrm{~mm}^{3}\right)$ from a single nude mouse was implanted by surgical orthotopic implantation (SOI) into the left lobe of the left lung in additional nude mice (group of eight animals). The animals were kept under isoflurane anesthesia during surgery. All procedures of the operation were performed with a $6.3 \times$ magnification microscope (Olympus, Tokyo, Japan), (Fig. 1).

Orthotopically-growing H460-RFP demonstrates aggressive metastatic behaviour [4]. Animal experiments were carried out in accordance with the Guidelines for the Care and Use of Laboratory Animals under NIH Assurance No. A3873-1.

Isolation and Culture of the Circulating Tumor Cells from Blood Blood (0.5-1.0 ml) was obtained from the nude mouse by cardiac puncture 1 month after orthotopic implantation of H460-RFP. The blood was placed in an EDTA tube (BD). A size-based separation method for viable CTC- enrichment (MetaCell ${ }^{\circledR}$, Ostrava, Czech Republic) by filtration of the peripheral blood (PB) through a porous polycarbonate membrane ( $8 \mu \mathrm{m}$ diameter pores) was used.

The membrane filters along with the plastic ring were transferred to 6-well tissue culture dishes (Fig. 1). RPMI 1640 medium $(4 \mathrm{ml})$ with $10 \% \mathrm{FBS}$ is added on top of the filter, and CTCs were then cultured on the membrane at $37^{\circ} \mathrm{C}$, $5 \% \mathrm{CO}_{2}$. After 14 days, the CTCs were cultured directly on the plastic dish surface or microscopic slides (Lab-Tek Chambered Coverglass, Thermo Fisher Scientific, Rochester, U.S.) for further confocal microscopy analysis.

Fluorescence Imaging The Olympus OV100 Small Animal Imaging System (Olympus Corp., Tokyo, Japan) was used for bright-field and fluorescence imaging of the mice with orthotopically-implanted H460-GFP. The OV100 has a sensitive CCD camera and four objective lenses, parcentered and parfo$\mathrm{cal}$, enabling imaging from macrocellular to subcellular [6].

Confocal Imaging A Leica (Wetzler, Germany) TCS SP5 AOBS confocal microscope was used along with a DFC350 FX Digital Camera for $72 \mathrm{~h}$ time-lapse imaging.

\section{Results}

CTC Isolation In the present report, we used a capillaryaction-driven size-based separation of the CTCs from the peripheral blood of orthotopic models of human tumors using the MetaCell ${ }^{\circledR}$ device. A schematic work- flow is shown on the Fig. 1. The CTCs were detected and separated in PB of all animals.

Due to the gentle blood flow within the separation process, caused by natural forces of capillary action, the enriched CTCs remained viable on the separating polycarbonate membrane (Fig. 2). The viability is evidenced by the expression of RFP-protein in the captured cells (Fig. 2b, c, d).

The CTCs enriched on the membrane, which is hold in a plastic ring, were then placed directly into a 6-well culture dish along with cell culture medium (Fig. 1). The cells were scanned every $12 \mathrm{~h}$ and the first mitoses have been observed after $24 \mathrm{~h}$ (Fig. 2c, d). Using this method, we obtained confluent H460-RFP CTCs culture within several days. The subsequent growth of confluent CTC-culture is shown on Fig. 3. Figure 3 is derived from time-lapse imaging.

CTC-Cytomorphology and Characteristics Generally, the cultured CTCs exhibited blebbing surfaces (Fig. 4a), the cell membrane vesicles (blebs) are exhibited already within 3 days of culture. Another typical characteristics of in vitro growing CTCs is multinuclear stages with prominent nucleoli (Fig. 4b).

The CTCs appeared very heterogeneous even if originating from one primary tumor. They differed in size $(11-30 \mu \mathrm{m})$ and proliferation capacity as evidenced by the time-lapse imaging (Fig. 5). Based on the time lapse imaging of the cells in vitro we may hypothesize that the cells use the blebs on the cell surface for faster movement if compared to the cells without blebbing. Morphologically, there is a difference between the CTC-culture and the original H460-RFP cell culture in the amount of produced blebs on the cell surface. The CTCs produce usually more blebs.

Following, irregular CTC-divisions were seen very often; when one CTC-cell divided into the 3 daughter cells (Fig. 6). The cell originating from these unbalanced divisions may harbor a potential to divide again into three daughter cells within the next mitotic cycle. As shown on the Fig. 6, some of the 3 daughter cells may enter apoptotic process immediately after division.

The uncontrolled growth of the CTCs population, with many irregular mitoses, is in fact generating a population of the cancer cells significantly different to the primary cell culture. The CTCs in vitro cultures are definitely worth to be studied subsequently by immunophenotypization, gene expression profiling and mutational analysis. 


\section{Orthotopic model of H460-RFP human lung cancer for CTCs testing}
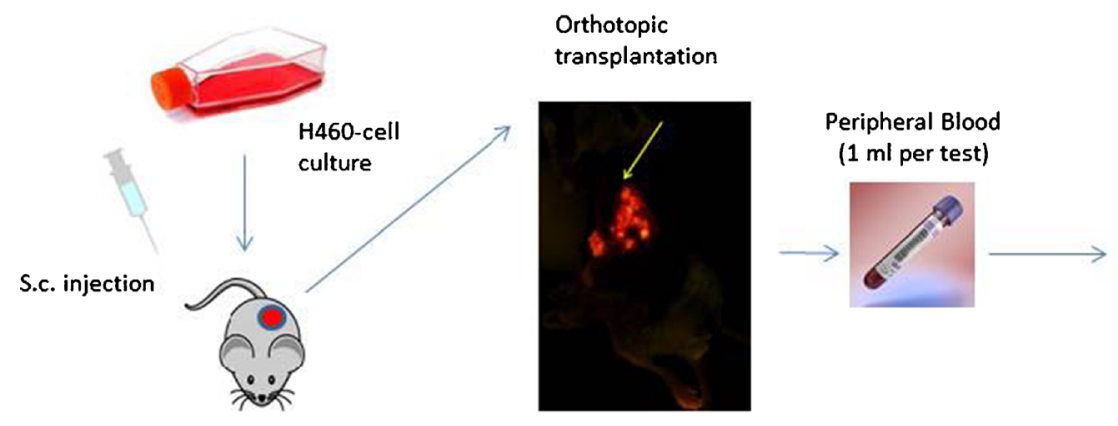
Capillary action driven CTCs separation

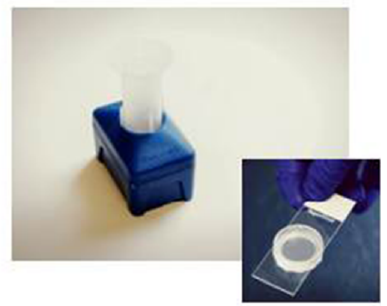

\section{CTCs in vitro culture ( Metacell ${ }^{\mathrm{TM}}$ )}
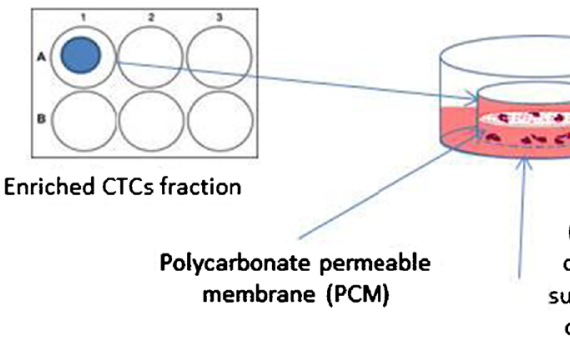

Growth Medium ( a slight flux driven only by diffussion is supported by the shape of the plastic holder)

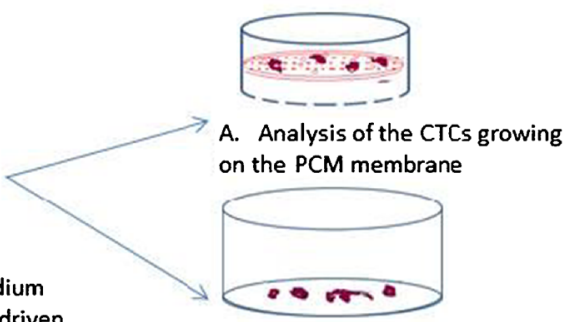

B. Analysis of the CTCs overgrowing the PCM membrane -* the bottom fraction

Fig. 1 Experimental design

Fig. 2 CTCs enriched from the peripheral blood (PB). PB was withdrawn from a mouse bearing H460-RFP human lung cancer growing orthotopically, 1 month after implantation. CTCs were detected on the microporous polycarbonate filter membrane by RFP-fluorescence. a, b $6 \mathrm{~h}$ after blood withdrawal. c, d $24 \mathrm{~h}$ after blood withdrawal. The first mitoses are observed already after $24 \mathrm{~h}$. The CTCs were kept in RPMI medium with FBS. Scale bar $10 \mu \mathrm{m}$ (Leica confocal microscope TCS SP5 AOBS)
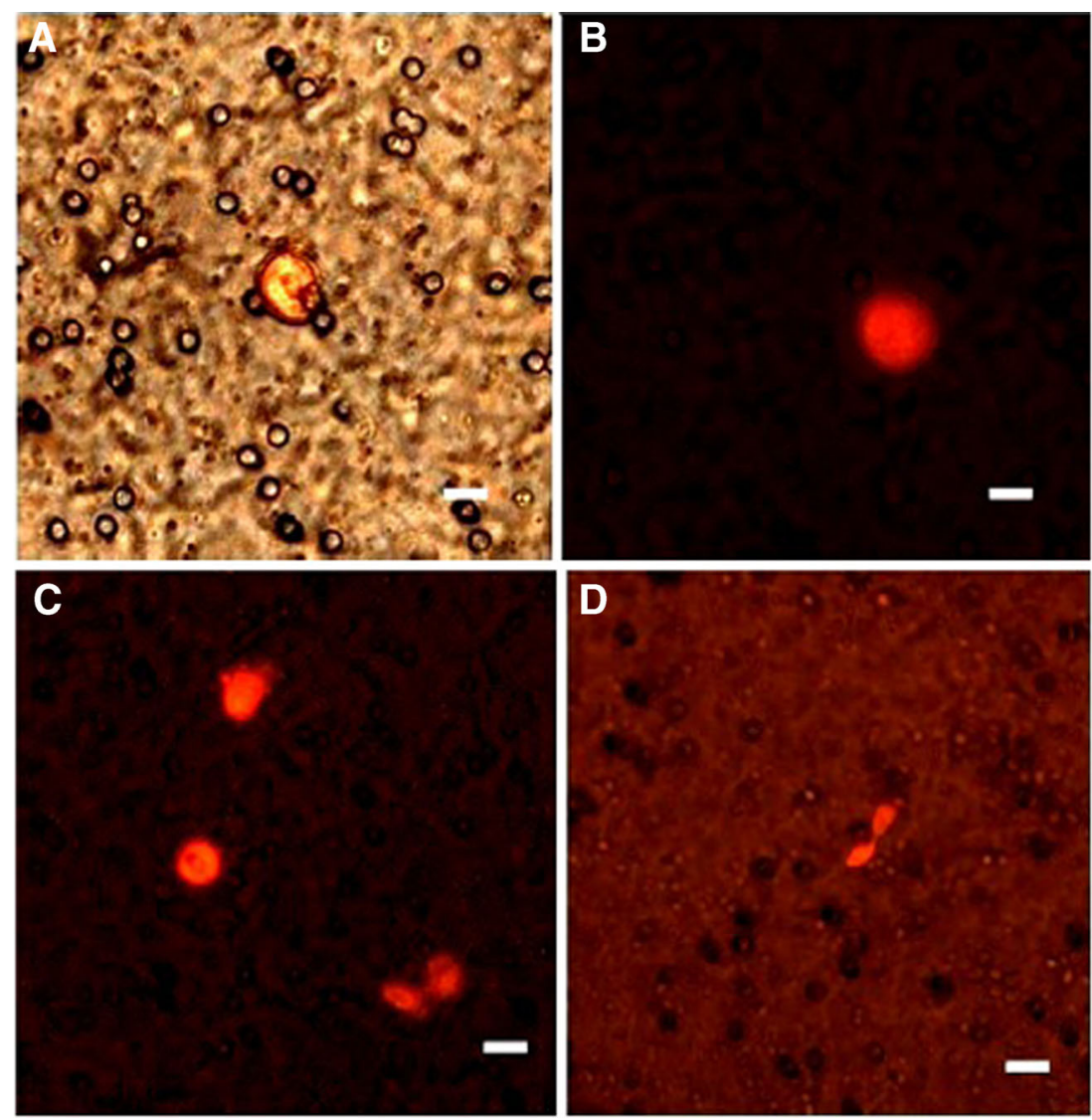

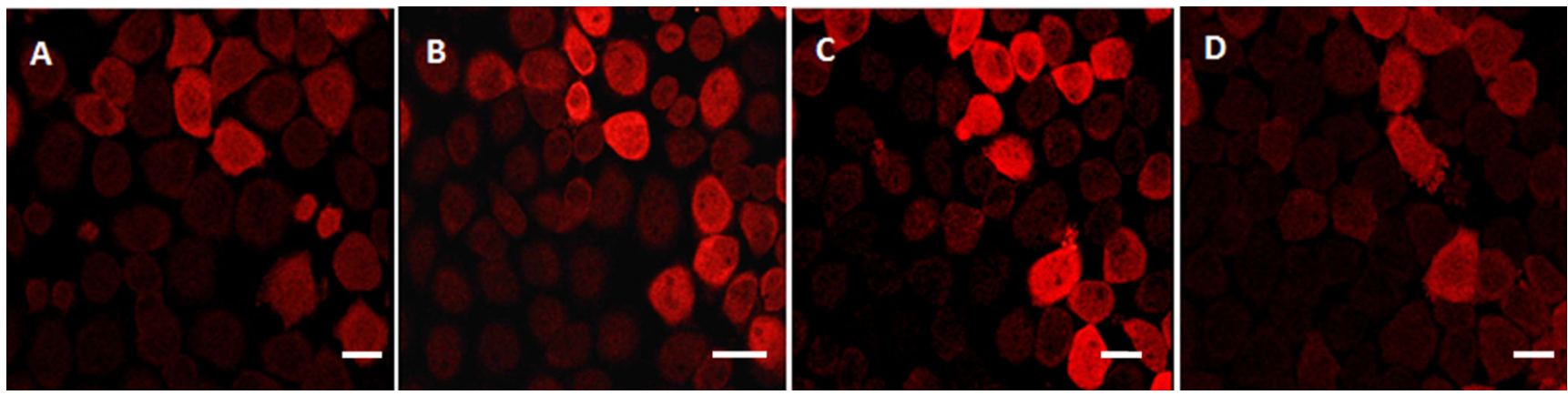

Fig. 3 CTCs isolated from a tumor bearing mouse (orthotopically-transplanted human H460-RFP), cultured in vitro. The subsequent growth of confluent CTC-culture is shown derived from time -lapse imaging within $72 \mathrm{~h}$. Scale bar $20 \mu \mathrm{m}$

\section{Discussion}

Our aim was to develop a successful CTC isolation method and CTCs in vitro culture protocol enabling cytomorphological analysis of viable CTCs. Cytomorphological characterization of CTCs can be followed by further downstream analysis (e.g., karyotype analysis, gene expression analysis of chemoresistance genes, in vitro chemoresistance monitoring). All the information on CTCs characteristics can be used for personalizing cancer treatment [7].

Previously, we have shown the feasibility of culturing CTCs directly from whole blood using orthotopic models of human tumors expressing GFP (green fluorescent protein) using immunomagnetic separation of the CTC cells [5].

In the present study, CTCs were isolated from the H460RFP orthotopic metastatic nude-mouse model of lung cancer by a size-based separation method. The majority of CTC isolation methods uses epithelial cell-adhesion molecule (EpCAM) to capture CTCs and cytokeratin (CK) antibodies to identify CTCs [8]. The weakness of these approaches could be that the rare cells isolated via EpCAM and/or cytokeratin (CK) binding may be circulating epithelial cells and more aggressive cell subpopulations might not have been isolated at all because they are EpCAM and CK negative $[9,10]$. There is a broad morphological and immunophenotypical variation within CTCs derived from the same tumor type as we have realized during our observation.

The EpCAM-dependance could be one of the reasons why significant differences in separation efficiency have been reported if the immunomagnetic isolation of lung cancer CTCs (CellSearch ${ }^{\circledR}$ ) was compared to size-based filtration CTCcapture (ISET ${ }^{\circledR}$ ) [11].

Therefore, accurate detection of CTCs based on morphological features such as size could be crucial. Since only a limited number of CTCs are captured by all, it is important to expand the isolated CTCs in vitro in order to perform subsequent functional analyses.

CTCs have been found to create doublets and clusters in the blood [12, 13]. Circulating tumor microemboli have been observed in the peripheral blood of metastatic lung cancer, as well $[11,14]$. The presence of clusters of CTC might be a relevant prognostic factor for malignancy [15]. However, large- and variable-sized clusters may not be isolated by immunomagnetic methods, since clusters may lack sufficient expression of EpCAM and/or CK. CTCs within clusters may also be surrounded by blood cells and thus, cannot be detected or recognized by immunogenic techniques $[14,16]$. An advantage of size-based separation methods could be enabling the cluster isolation as well.

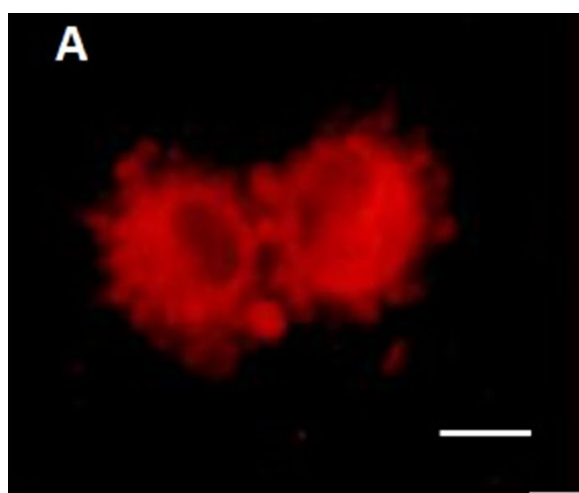

Fig. 4 CTC -proliferation evidenced in vitro, in the culture of CTCs, isolated from an H460 experimental human lung cancer mice model. a Cell membrane vesicles starting to be produced within 3 days of culture. b

\section{B}

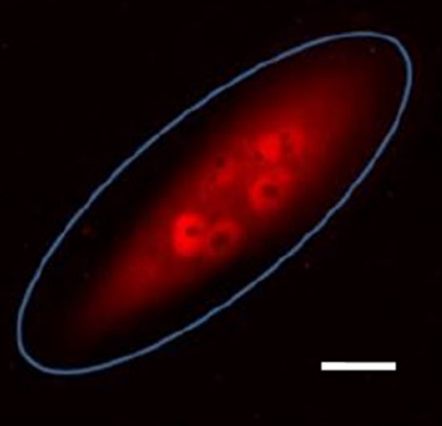

Multi-nuclear stage can be also found very often in the CTCs. The CTCs were kept in RPMI medium with FBS. Scale bar $10 \mu \mathrm{m}$ (Leica confocal microscope TCS SP5 AOBS) 


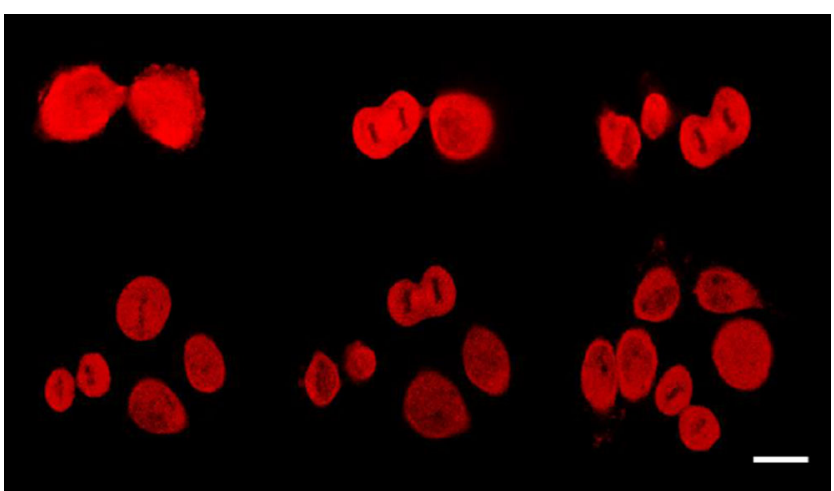

Fig. 5 CTC proliferation monitored in vitro, in the culture of CTCs isolated from an H460 experimental human lung cancer mice modelconfocal time-lapse imaging. In vitro culture was started from a single CTC-cell. One mitotic cycle took approximately $12 \mathrm{~h}$ in average. Scale bar (Leica confocal microscope TCS SP5 AOBS)

But there are also differences in the capture efficiency for the recently introduced size- based separation methods (e.g. ISET ${ }^{\circledR}, \mathrm{ScreeCell}^{\circledR}, \mathrm{CellSieve}^{\circledR}$, MetaCell ${ }^{\circledR}$ ). So far no comparison study has been conducted in the field of sizebased separation methods. The most important difference between the reported size-based separation methods is that not all of them enable to capture viable CTCs.

On the other hand methodologies reporting viable CTCcells enrichment have also not shown until today, how many out of the captured CTCs do survive the isolation process and are able to proceed towards mitotic process. The analytical reports evidencing the CTC- viability are generally based on the isolation of the cancer cells out of cell cultures diluted in the blood samples. One could expect, that these cells will behave differently, than CTCs isolated from patients' blood or animal model.

We believe that data presented in this article, based on the use of orhotopic metastatic models, could be an unique in vivo proof of the viable CTCs separation principle.

One could hypothesize, that the use of orthotopical metastasis models could enhance the validation process for the size-based separation methods, in general. Having more viable CTCs leads us faster towards personalized cancer treatment.

We hope that combination of SOI technique with a use of cancer cell lines expressing fluorescent protein and subsequent detection of CTCs/clusters by size-based separation method (MetaCell ${ }^{\circledR}$ ) could provide easier way for new anticancer agents testing. The count of CTCs appears to monitor the response to the treatment, in as little as a few weeks $[13,16]$. Beyond an in vitro number count, an ex vivo functional study on patient-derived CTCs might provide a support for an immediate treatment decision regarding drug resistance [11].

In vitro culturing of CTCs is a base for the proliferation tests comparing the chemosensitivity. This study demonstrate a successful culturing of CTCs isolated from animal using filtration device $\left(\mathrm{MetaCell}^{\circledR}\right)$, which is as gentle as enabling obtaining CTCs with very high viability. These protocols can focus on using the CTCs culture for personalizing the oncological treatment in future.
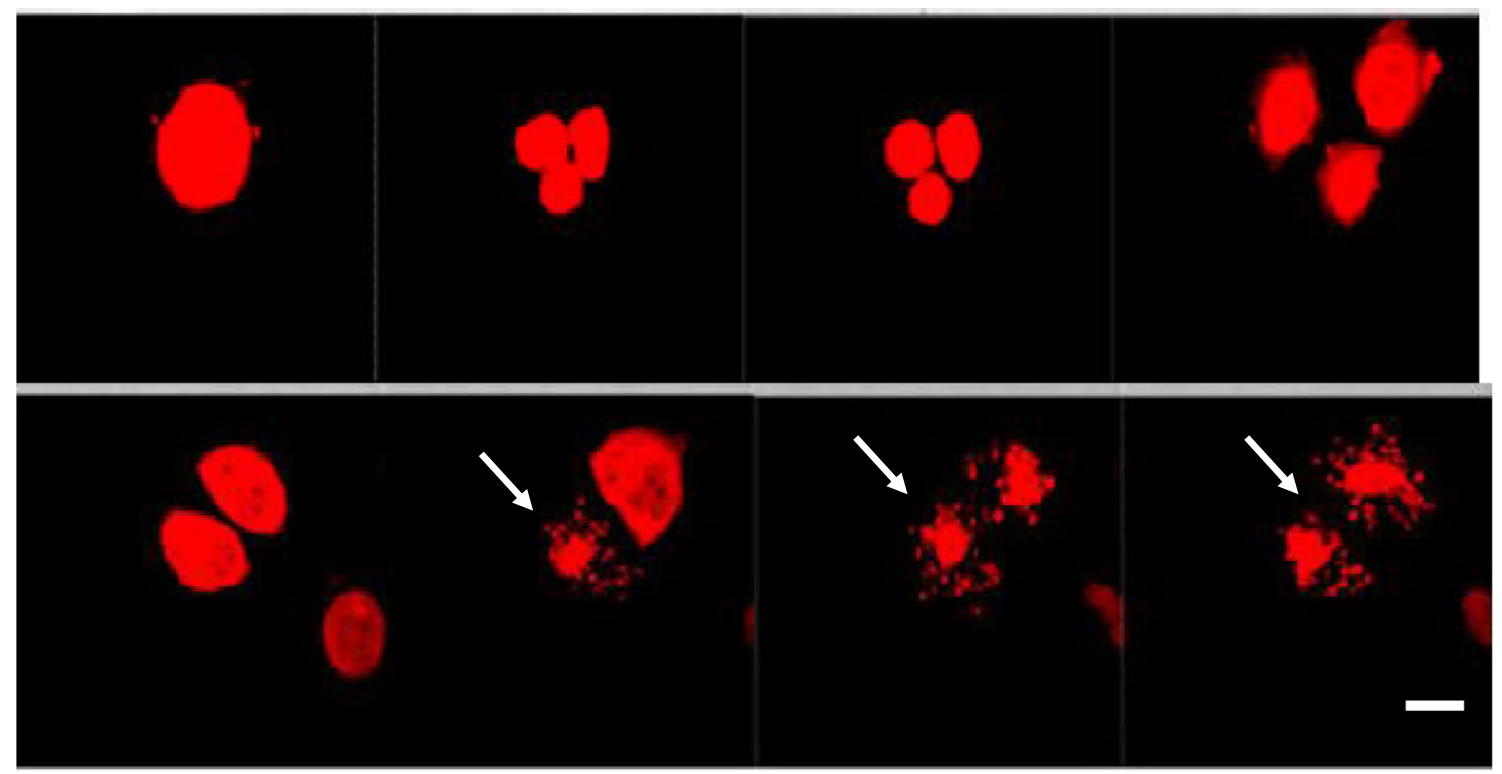

Fig. 6 CTCs isolated from a tumor bearing mouse (orthotopicallytransplanted human H460-RFP), cultured in vitro, imaged by time lapse imaging for $72 \mathrm{~h}$. An irregular mitosis of one dividing cell (in the multinuclear stage) is shown on the picture. Subsequently three daughter cells developed. Each daughter-cell divided within the next mitotic cycle into 3 cells. Afterwards an apoptosis process started (see arrows). The CTCs were kept in RPMI medium with FBS. Scale bar $10 \mu \mathrm{m}$ (Leica confocal microscope TCS SP5 AOBS) 


\section{Conclusion}

In vitro culturing of CTCs is a base for the proliferation test reporting the chemosensitivity. This study demonstrate a successful culturing of animal CTCs by use of filtration device (MetaCell $\left.{ }^{\circledR}\right)$, which is as gentle as enabling obtaining CTCs with very high viability. These protocols can focus on using the CTCs culture for personalizing the oncological treatment in future.

Acknowledgments Supported by the research project conceptual development of research organization, University Hospital Motol, Prague Czech Republic, 00064203 and Research project P 27/2012 awarded by Charles University in Prague

Conflict of Interest The authors report no conflicts of interest.

Open Access This article is distributed under the terms of the Creative Commons Attribution License which permits any use, distribution, and reproduction in any medium, provided the original author(s) and the source are credited.

\section{References}

1. Hong B, Zu Y (2013) Detecting circulating tumor cells: current challenges and new trends. Theranostics 3(6):377-394. doi:10. 7150/thno.5195, Print 2013

2. Alunni-Fabbroni M, Sandri MT (2010) Circulating tumour cells in clinical practice: methods of detection and possible characterization. Methods 50(4):289-297

3. Paterlini-Brechot P, Benali NL (2007) Circulating tumor cells (CTC) detection: clinical impact and future directions. Cancer Lett 253(2): 180-204

4. Yang M, Hasegawa S, Jiang P, Wang X, Tan Y, Chishima T, Shimada H, Moossa AR, Hoffman RM (1998) Widespread skeletal metastatic potential of human lung cancer revealed by green fluorescent protein expression. Cancer Res 58(19):4217-4221
5. Kolostova K, Pinterova D, Hoffman R, Bobek V (2011) Circulating human prostate cancer cells from an orthotopic mouse model rapidly captured by immunomagnetic beads and imaged by GFP expression. Anticancer Res 31:1535-1540

6. Yamauchi K, Yang M, Jiang P et al (2006) Development of real-time subcellular dynamic multicolor imaging of cancer cell trafficking in live mice with a variable-magnification whole-mouse imaging system. Cancer Res 66:4208-4214

7. Zhou J, Hu L, Yu Z et al (2011) Marker expression in circulating cancer cells of pancreatic cancer patients. J Surg Res 171: 631-636

8. CellSearch (2012) Circulating Tumor Cell Epithelial Kit (IVD), CellSearch ${ }^{\circledR}$ Instructions for Use (IFU), No. e631500023. CellSearch

9. Attard G, de Bono JS (2011) Utilizing circulating tumor cells: challenges and pitfalls. Curr Opin Genet Dev 21:50-58

10. Marrinucci D, Bethel K, Kolatkar A et al (2012) Fluid biopsy in patients with metastatic prostate, pancreatic and breast cancers. Phys Biol 9(1):016003

11. Hou JM, Krebs M, Ward T et al (2011) Circulating tumor cells as a window on metastasis biology in lung cancer. Am J Pathol 178:989-996

12. Allard WJ, Matera J, Miller MC, Repollet M, Connelly MC, Rao C, Tibbe AG, Uhr JW, Terstappen LW (2004) Tumor cells circulate in the peripheral blood of all major carcinomas but not in healthy subjects or patients with nonmalignant diseases. Clin Cancer Res 10(20):6897-6904

13. Christiansen JJ, Rajasekaran AK (2006) Reassessing epithelial to mesenchymal transition as a prerequisite for carcinoma invasion and metastasis. Cancer Res 66(17):8319-8326

14. Hou JM, Krebs M, Ward T, Morris K, Sloane R, Blackhall F, Dive C (2010) Circulating tumor cells, enumeration and beyond. Cancers 2(2):1236-1250

15. Vona G, Estepa L, Béroud C, Damotte D, Capron F, Nalpas B, Mineur A, Franco D, Lacour B, Pol S, Bréchot C, PaterliniBréchot $P$ (2004) Impact of cytomorphological detection of circulating tumor cells in patients with liver cancer. Hepatology 39(3): 792-797

16. Deng G, Herrler M, Burgess D et al (2008) Enrichment with anti-cytokeratin alone or combined with anti-EpCAM antibodies significantly increases the sensitivity for circulating tumor cell detection in metastatic breast cancer patients. Breast Cancer Res 10:R69 